\section{Restrictions and arbitrary rules}

PALABRAS CLAVE I RESTRICCIÓN I MANUAL | PROCESO DE PALABRAS CLAVE | RESTRICCION | MANUALL | PROCESO DE
DISENNO | INVESTICACIÓN POR DISEÑO | REGLAS ARBITRARIAS KEYWORDS | RESTRICTION | MANUAL | DESICN PROCESS | RESEARCH BY DESICN I ARBITRARY RULES

\section{| RESUMEN |}

¿Es el diseño arquitectónico un proceso lineal? ¿puede anticiparse el diseño a partir de fundamentos iniciales fijos? Si consideramos que el diseño arquitectónico es una forma de investigación, en la que jamás se pueden anticipar los accidentes y descubrimientos del proceso, podemos sospechar que las prescripciones argumentales iniciales no podrán mantenerse invariables hasta el estado final de un proyecto. A partir de la experiencia académica del taller de proyectos "El Diccionario de Ideas recibidas" se plantea un análisis acerca de la utilidad de los argumentos iniciales de proyecto, no para definir un diseño deseable, sino para generar un marco operativo de límites y reglas que nos alejan intencionalmente de esta condición deseable o conocida, y cuyo planteamiento arbitrario reafirma su utilidad como herramienta de desapego respecto de dichas preconcepciones.

\section{| ABSTRACT |}

Is architectural design a linear process? Can initial arguments foresee design? If we consider architectura design as a type of research that cannot anticipate accidents and discoveries of the process, we may thus suppose that the starting argumentative prescriptions cannot stay unchanged up to the end of a project. From the academic experience of the architectural design studio "The Dictionary of Received Ideas", I analyze the usefulness of the starting project arguments that fail to define a desirable design object, but generate an operative framework of rules and boundaries that intentionally separates it from this desirable or recognizable condition. The arbitrary terms of this framework restates its utility as a tool for detachment from said preconceptions.

\title{
Restricciones y arbitrariedades
}

\author{
CLAUDIO PALAVECINO LLANOS*·Universidad de Chile, Chile·cpalavecino@uchilefau.cl
}

Fecha de recepción 21/enero/2015 - Fecha de aceptación 03/junio/2015

\begin{abstract}
Mark Wigley -ex decano de la Escuela de Arquitectura de la Columbia Universitysolía decir que "un arquitecto es alguien que sabe muchas cosas, excepto lo que es un edificio". Parece poco cuestionable que los arquitectos somos expertos en diseñar edificios, pese a que existe poca claridad acerca de cómo diseñamos, y no parece que queramos sistematizar este conocimiento experto para reproducirlo nuevamente. Lo que entendemos por pericia en la arquitectura, al parecer no significa manejar un gran acervo de conocimiento sobre cómo diseñar eficaz y exitosamente varios tipos de edificios -si es que se puede sistematizar algo así-, sino una cierta flexibilidad para transar entre lo que el diseñador desea y lo que el medio condiciona, obteniendo idealmente el beneficio de todas las partes. Pareciera ser que la calidad de un proyecto no depende solo de la habilidad y los argumentos del arquitecto a cargo, sino de una negociación entre estos y una realidad que puede ser arbitraria, cambiante, ambigua y en ocasiones paralizante.
\end{abstract}

Si bien es cierto que casi todos los arquitectos son conscientes de esta condición, es paradójico ver cómo en la enseñanza de la arquitectura predomina la correspondencia entre las premisas argumentales iniciales y la propuesta como fin último de evaluación. Esta noción supone que si existe claridad en las premisas iniciales, estas guiarán el desarrollo del proyecto hasta el final, siendo el diseño resultante una respuesta al problema inicial. Según esta lógica, la ausencia de argumentos claros en un inicio, impide visualizar el rumbo que tomará el proyecto, y las constantes variaciones durante el proceso reflejan poca claridad en lo que se quiere e inconsistencia en la metodología de trabajo, siendo el diseño final el resultado de una dinámica de prueba y error sustentado en las calificaciones (notas) del proceso. El objetivo de esta dinámica es acertar a una configuración de argumentos que puedan proyectarse en un futuro diseño, sin tener que imponer cambios o correcciones bruscas durante el resto del proceso.

En el medio académico se han establecido numerosos sistemas que incentivan la construcción de argumentos como si se trataran de planes que deben ser ejecutados a través del diseño. Estos suelen sintetizarse en conceptos

\footnotetext{
Arquitecto de la Universidad de Chile y Master in Advanced Architectural Design de la Columbia University. Ha desarrollado su profesión en algunas de las más importantes oficinas de Chile. Sus proyectos e iniciativas han sido premiadas en concursos y seleccionadas para publicaciones y exposiciones en Chile, Europa y Norteamérica. Luego de sus estudios en los EE.UU., ha desarrollado la docencia junto a Alberto Fernández, dictando el Taller de Diseño Arquitectónico en la Facultad de Arquitectura y Urbanismo de la U. de Chile. Actualmente dirige el proyecto de publicación, difusión y estudio de proyectos de arquitectura de pregrado "Proceso".
} 
-representación de un fenómeno o condición a través de una palabra, frase o abstracción gráfica-, ideas fuerza, imágenes objetivo generar imágenes que evoquen un resultado deseable-o lineamientos obtenidos a partir de un análisis o estudio previo, en donde la propuesta (edificio, prototipo, plan urbano, etc.) es una solución a un problema reconocido. Estas acciones definen al argumento como un ente rector que discrimina lo correcto de lo incorrecto. Así, mientras mayor coherencia exista entre este argumento y el diseño, mejor será el resultado.

Esta concepción de diseño considera que el fundamento inicial es un indicador del resultado final o que al menos, el éxito del diseño final podría anticiparse a partir de las primeras propuestas, y que por lo tanto existiría un espectro restringido de resultados correctos o aceptables según la problemática tratada. Esta escala de valoración surge de la similitud entre el ejercicio de diseño y otras experiencias proyectuales exitosas (sobre todo casos contemporáneos, conocidos y validados por el medio), asumiendo que mientras más proximidad exista entre lo sugerido y la referencia, más posibilidades existen de que se repita el éxito. Dicho de otra forma, el proceso de diseño así entendido, no tiene más alcance que corroborar un conocimiento preconcebido.

Si bien estas formas de aproximarse al proyecto permitirían a los futuros arquitectos adquirir una metodología funcional de trabajo -al menos para el medio laboral actual- esta rechazaría el potencial creativo de la profesión. El diseño arquitectónico es una labor intelectual que genera conocimiento, una actividad creativa en la cual el resultado es desconocido, ya que persigue preguntas cuyas respuestas no están definidas. Dicho ejercicio rara vez (me atrevería a decir nunca) es un proceso lineal, que considera reformular las certezas iniciales, probar y evaluar resultados, retroceder en ciertas etapas de trabajo y asumir el diseño resultante como aprendizaje desde el momento en que sobrepasa las expectativas iniciales, alejadas de los parámetros simplificados de éxito o fracaso.
¿Hasta qué punto es posible planificar un proceso de diseño?, ¿̇pueden los argumentos e intenciones del diseñador guiar el proceso de diseño de un proyecto? Podríamos considerar el fundamento principal de un proyecto como un punto de inicio, una conclusión o un cuerpo flexible de argumentos que cambia en la medida que el diseño lo hace, que se reescribe en la medida en que interpreta con precisión el resultado proyectual como descubrimiento y no como una constatación forzada de una premisa inicial, ya que el diseño -como proceso de investigación- no debiese estar necesariamente dirigido o predestinado. De manera más precisa, asumir el proceso de diseño como un camino de accidentes que obliga a redirigir estrategias y redefinir los límites iniciales, es valorizar el diseño como ejercicio de investigación.

¿Cómo se pueden forzar los accidentes o los descubrimientos de un proceso de diseño si estos no pueden anticiparse? Si entendemos que la imposición de argumentos al proyecto fuerza un resultado, al extremo de rechazar los posibles accidentes del proceso que desestabilizan la correspondencia entre inicio y final, podríamos considerar en primera instancia, que el diseñador operase en un marco en el que los argumentos no necesariamente tienen que regir el diseño esperable, sino que pueden constituir un sistema de trabajo o metodología en donde el resultado es incierto. Es decir, los argumentos iniciales no anticipan el proyecto, sino que generan un marco de operaciones para que este surja, no como una preconcepción, sino como un descubrimiento.

\section{EL DICCIONARIO DE IDEAS RECIBIDAS}

Recientemente he podido estudiar metodologías de trabajo basadas en la autoimposición de restricciones, que definen una serie de planteamientos fijos sobre cómo trabajar el diseño que debe ponerse a prueba a través del proyecto. Si bien, todo proyecto de arquitectura debe enfrentar muchas restricciones que surgen de fundamentos y demandas externas al desarrollo de un proyecto, tales como plazos, costos, condiciones impuestas por mandantes o personal de trabajo; estas son comúnmente entendidas como obstáculos, porque al ser abordadas no permiten la resolución óptima del diseño. Debido a que muchas de estas imposiciones son normalmente anticipables, las posibles soluciones a estas suelen ser igualmente anticipables, repitiendo fórmulas y estrategias con mayor o menor espacio para generar cambios.

Las restricciones autoimpuestas serían nuevas medidas arbitrarias decididas por el arquitecto o el equipo de trabajo, alejada de los requisitos externos ya mencionados. Estas no tienen una relación directa con el proyecto, pero si lo tienen con la metodología que lo determina, las preguntas y problemas fundamentales que definen la matriz de diseño. Al operar fuera de los márgenes tradicionales de las imposiciones externas, el diseño diverge hacia vías inexploradas de trabajo, accidentes y escenarios inesperados que permiten desapegarnos de los lugares comunes del diseño arquitectónico, y generar innovaciones que permiten "desatar el potencial del proyecto" (Walker, 2013). Estas restricciones son un medio que permite despegarnos de lo conocido o lo asumido acerca del proyecto.

Esta premisa fue la base de un taller de proyectos del cual fui parte (entiéndase "taller de proyectos" como convención para llamar a la cátedra "studio" enfocada a desarrollar proyectos a partir de una tesis), llamado "El Diccionario de Ideas Recibidas" por su instructor, el profesor Enrique Walker en la Columbia University. El taller tiene como premisa el texto homónimo de Gustave Flaubert, un compendio de clichés de la sociedad francesa del siglo XIX recopiladas durante la mayor parte de la vida de este autor, y que dejaba en evidencia como el uso reiterativo de ciertas expresiones, lugares u objetos generaba un agotamiento de su valor original, convirtiéndolos en clichés (Walker, 2010). De la misma forma que Flaubert, el taller tendría como objetivo explorar métodos y operaciones conceptuales alternativas de diseño de proyectos, reconociendo una serie de clichés arquitectónicos como punto inicio. 


\section{EL MANUAL DE ARQUITECTURA ADORABLE}

La primera etapa de trabajo del taller consistió en reconocer clichés en proyectos y obras contemporáneas. Estos podían referirse a operaciones de diseño, tecnologías, materiales, lenguajes formales, configuraciones programáticas, estrategias de distribución espacial o incluso medios expresivos. A diferencia de los talleres de años anteriores, que habían abordado una serie de clichés como cuerpo de estudio, en esta ocasión abordamos un solo gran cliché, que denominados como "arquitectura adorable" - "Cute"-. Este se basaba en ciertas tendencias de la arquitectura japonesa de los últimos años para crear edificios que provocaran una sensación de fragilidad, limpieza y ascetismo extremo, ausencia manifiesta de uniones y elementos de encuentro entre piezas constructivas, gran simpleza formal y composición de vanos y muros que enfatizan la pequeñez, eliminando todo rasgo de pesadez y masividad de los grandes edificios. En esta etapa no existía una claridad evidente de qué era o no era un edificio "adorable", y por esa razón, inventariamos un sistema de reglas que definiera este cliché con precisión.

Estas reglas estarían contenidas en un manual, similar a un instructivo de ensamblaje, que en teoría permitiría producir un "proyecto adorable" si se siguen correctamente las instrucciones. A excepción de la mayoría de las indicaciones iniciales que se entregan un taller de proyectos, que consideran objetivos generales y limitantes de proyecto, como programas, usos o localización; el manual establece varias restricciones específicas sobre el diseño arquitectónico y define con extrema precisión qué tipo de elementos se deben usar, bajo qué condiciones estos son válidos y bajo cuáles no, así como sobre qué principios se han establecido dichas operaciones.

Las reglas escritas en este manual fueron decididas democráticamente por todos los integrantes del taller, y corregidas varias veces hasta tener un instructivo carente
1. Síntesis de operaciones del "Cute Manual" (Manual de Arquitectura Adorable).

Créditos: Laura Buck, Rui Wang, Myung Shin Kim, Alejandro Stein, Esteban de Backer, Ignacio Urquiza, Rodrigo Valenzuela, Nicolo Lewanski, Jordan Anderson, Della Leapman, Robert Morgan y Claudio Palavecino.

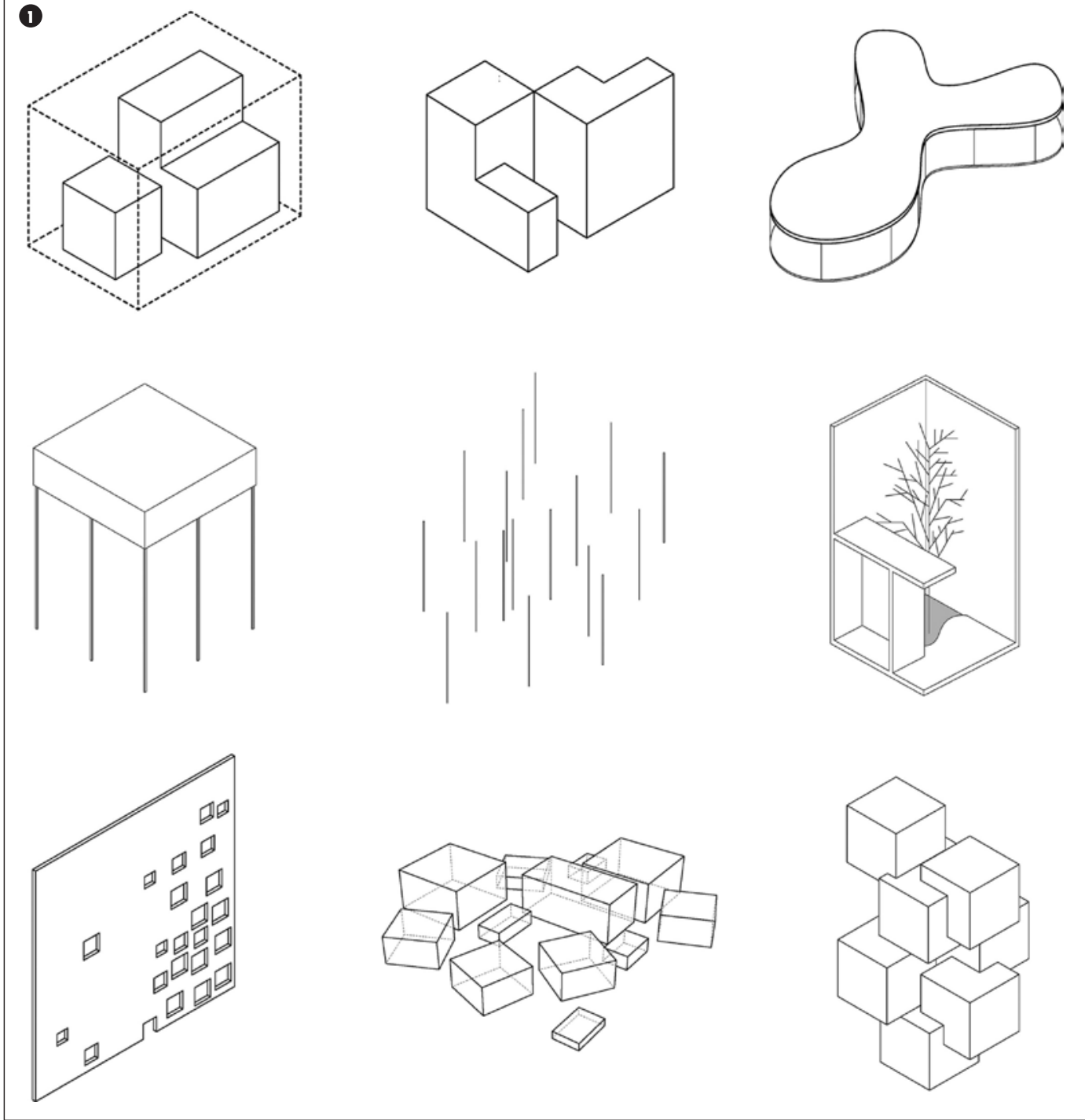

de ambigüedades, que no permitiese más interpretaciones que las estrictamente descritas y que fuera totalmente funcional para empezar a trabajar. De forma consciente, el manual fue redactado solo considerando la fidelidad de las reglas de diseño respecto del cliché en estudio, evitando previsualizar cualquier posible resultado de diseño durante su elaboración. De esta forma, no se dispone de condiciones habituales o al menos fáciles de manejar para generar proyectos -asumiendo que dichas condiciones dan más libertad creativa, o que al menos permiten vislumbrar posibles resultados satisfactorios, homologando dichas condiciones con antecedentes de proyectos anteriores-, sino que se extraen los límites del cliché para imponer un marco teórico y práctico de diseño que obligue a los diseñadores a operar fuera sus convenciones habituales, en la que la única forma de llegar a un resultado es a través del manual, ya que sin este no se puede ejecutar nada. 
2. Extracto de páginas del "Cute Manual" (Manual de Arquitectura Adorable).

Créditos: Laura Buck, Rui Wang, Myung Shin Kim, Alejandro Stein, Esteban de Backer, Ignacio Urquiza, Rodrigo Valenzuela, Nicolo Lewanski, Jordan Anderson, Della Leapman, Robert Morgan y Claudio Palavecino.

(2)

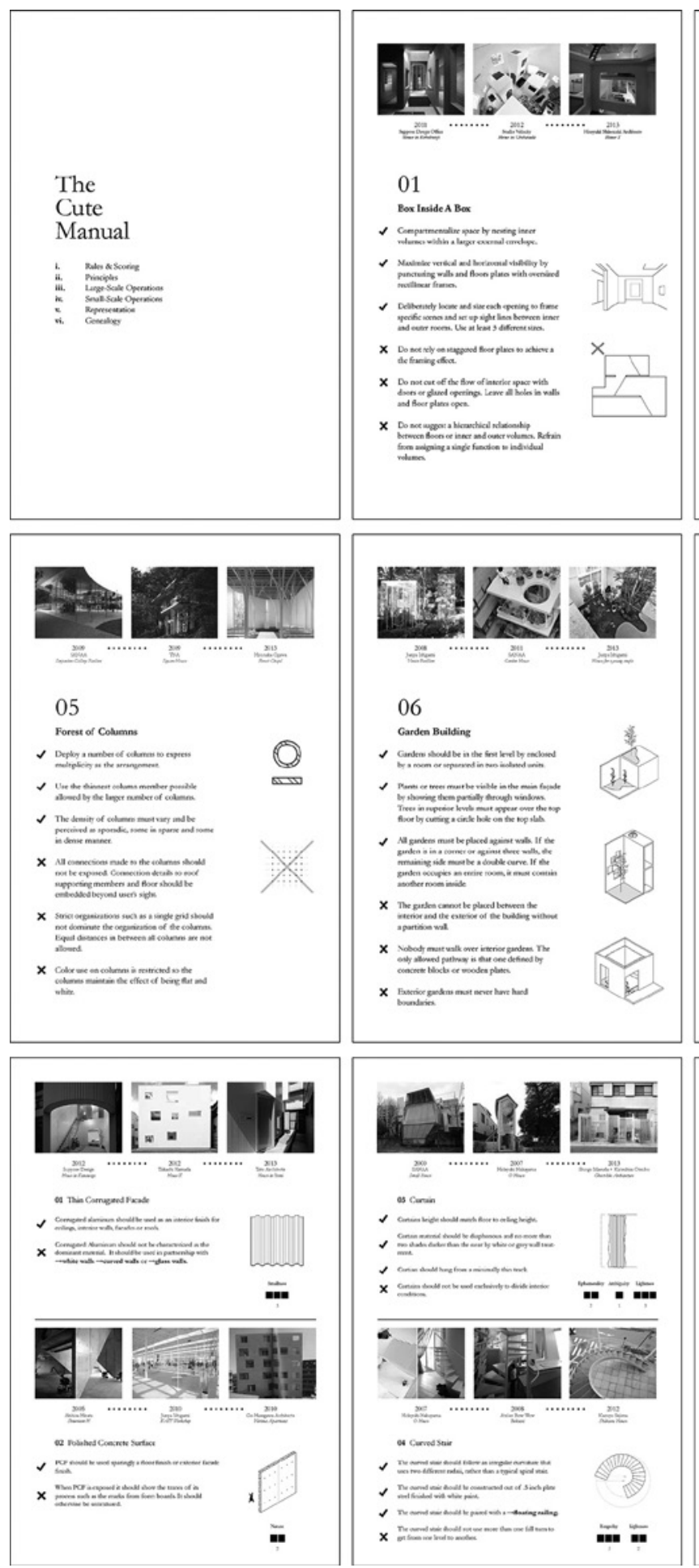

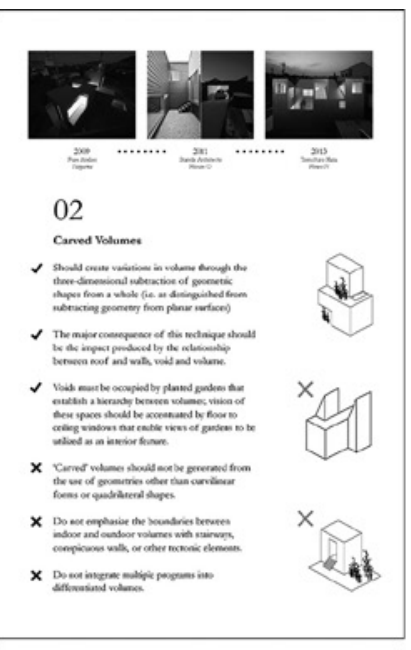

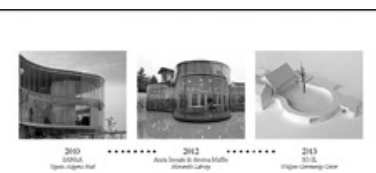

03
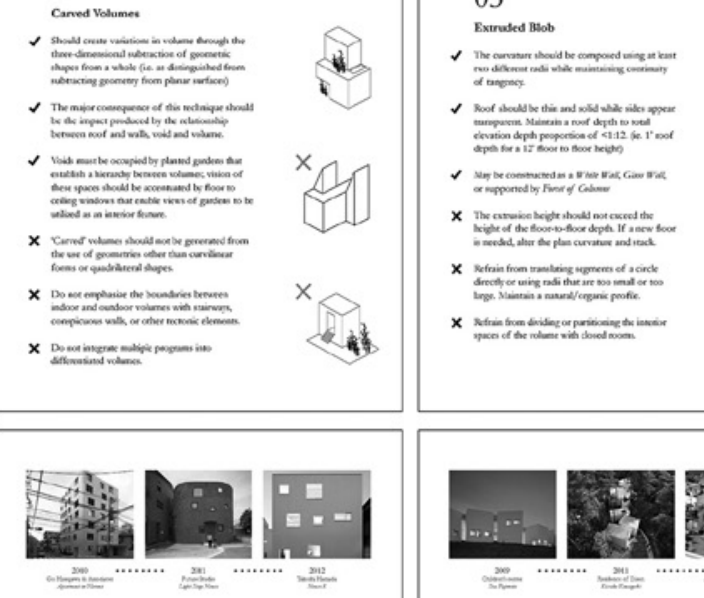

07

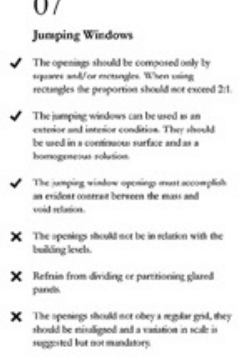

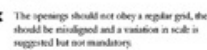

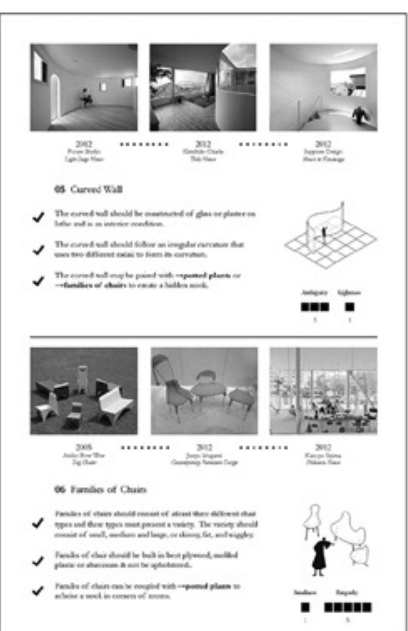

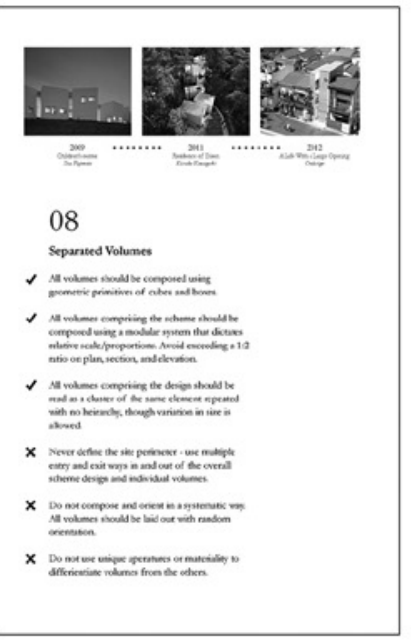

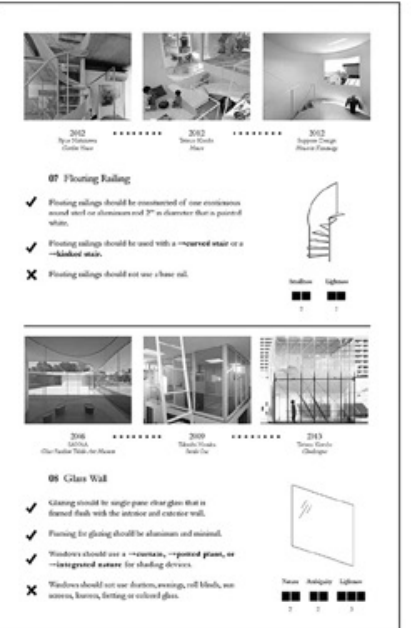

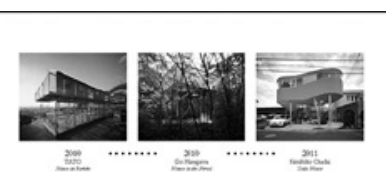

04

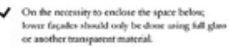

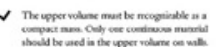

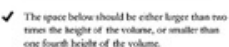

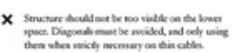

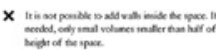

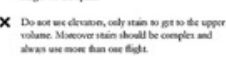

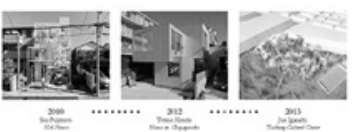

09

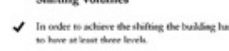

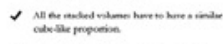

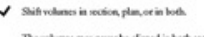

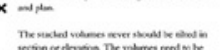

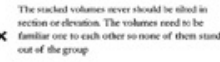

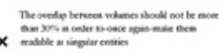

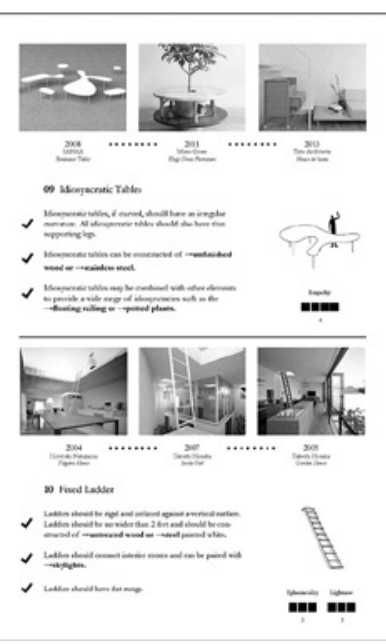


3. Diagrama de componentes proyecto "The Flower", estudio "El Diccionario de Ideas Recibidas". Créditos: Jordan Anderson, Della Leapman, Robert Morgan y Claudio Palavecino.

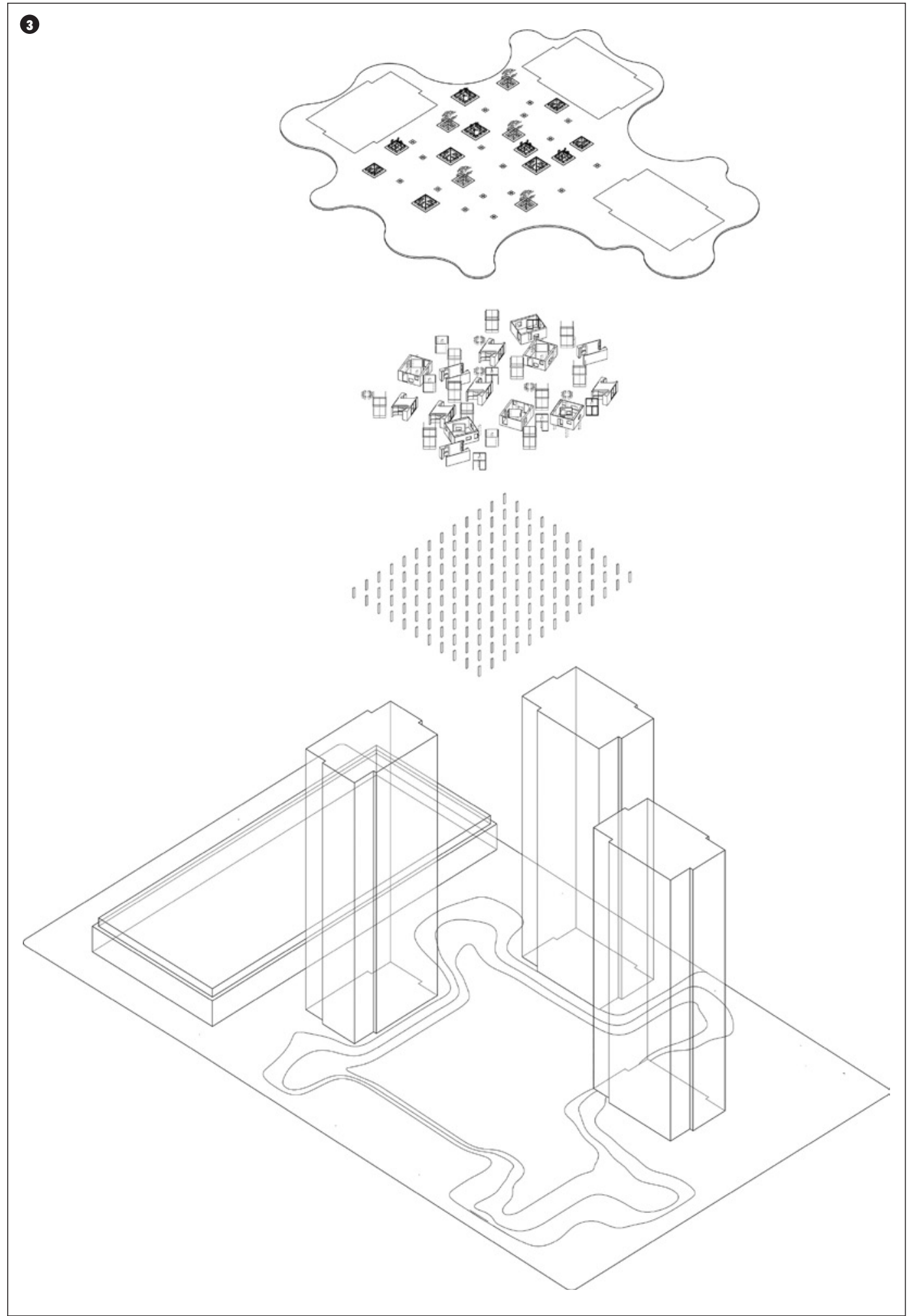

Dichas reglas estaban sometidas a seis principios básicos - pequeñez, materialidad efímera, relación con la naturaleza, ambigüedad, empatía y ligereza- que representaban los ideales que justifican cada operación de diseño para lograr materializar el cliché. Las reglas de diseño estaban puntuadas según su fidelidad con cada uno de los principios inicialmente definidos. De esta forma, cada proyecto consideraba una cantidad limitada de operaciones de diseño posible.

Las operaciones de diseño fueron nombradas de acuerdo a la manera en que se implementaban en los proyectos japoneses que ineludiblemente hacían referencia a estos ideales de la "arquitectura adorable". Las operaciones de gran escala estaban dirigidas a configurar los elementos de obra gruesa del proyecto, y consideraban algunas como la "caja dentro de la caja" que mostraba una tendencia a generar volúmenes ortogonales en el espacio interior aislados de la envolvente; "flor" que definía las curvatura de muros y tabiques evocando la silueta de una flor de loto visualizada en planta; "bosque de columnas" que repartía la cargas verticales en una gran cantidad de pilares muy esbeltos distribuidos fuera de la estructura de grilla; o "ventanas saltarinas" alusivo a la distribución de pequeñas ventanas cuadradas libremente dispersas en un muro, entre otras. Cada una de estas operaciones estaba definida por reglas sobre qué acciones seguir y cuáles no, una imagen general que sintetizaba estas reglas y un conjunto de imágenes de proyectos referentes.

Las operaciones de pequeña escala hacían referencia al uso de materiales, terminaciones y mobiliario, dentro de las que se incluyeron reglas para la curvatura de escaleras, uso de muros blancos y curvos, ventanas cuadradas en los cielos, entre otros. Finalmente, se incluyó un conjunto de reglas de representación específicas sobre ciertos elementos de dibujo, uso de colores, detalles planimétricos, líneas y otros recursos gráficos y de modelado. 
En síntesis, el manual sistematiza y organiza una serie de reglas con mucha precisión sobre aspectos de diseño que normalmente no son tratados con este grado de rigidez, ni mucho menos puntuado e inventariados en un manual. En efecto, ver que reglas de diseño son puntuadas numéricamente, y que dicho valor indica que tan "adorable" es el proyecto resultante parecería más una sátira de ciertos paradigmas de la arquitectura definidos por principios absolutos y manifiestos, pero este enfoque adoptado para su elaboración tenía un trasfondo mucho más experimental. Esto se reconoce primero, porque se planteó la elaboración del manual de forma similar a como se redactan las reglas de un juego, no solo por que estas fueran lo suficientemente precisas como para evitar trampas, sino porque no planteaban una manera de hacer proyectos mejores o más productivos, sino más bien una suerte de divertimento al reconocer la persistente presencia del cliché en obras y la formalización de este en reglas. Por otra parte, existía una incógnita total de qué proyectos podían surgir a partir del uso del manual, ya que si bien este nos debía conducir mecánicamente a diseñar edificios que pudiesen entenderse como "adorables", el hecho de conjugar métodos de diseño propios, convenciones culturales distintas a las que definieron el cliché y ponerlos en la perspectiva del manual, descomponía esta noción inicial.

Más allá de la experiencia propia de este taller, el manual de clichés era una herramienta que ya se había utilizado anteriormente en esta instancia, y cumplía la formalidad de nombrar y explicar las reglas del juego que debíamos seguir. Esto consiste en transparentar cómo muchas de nuestras decisiones como diseñadores reproducen ideas exitosas previamente aplicadas, no para generar un avance o mejora en el diseño, sino para replicar el éxito de su aplicación original. El manual así ofrece todas las condiciones para hacer un proyecto deseable y potencialmente exitoso (si es que consideramos exitoso el ser aceptado en la sociedad, como lo indicó Flaubert al justificar la existencia de los clichés sociales en su "Diccionario"), pero al hacerlo se consuman una serie de ideas agotadas y que irían en contra del valor de la propuesta de arquitectura como aporte o innovación.

\section{DISEÑAR CON UN MANUAL}

Los proyectos del taller fueron desarrollados por equipos de diseño. Cada equipo era libre de decidir la tipología, destino, tamaño o configuración programática de sus proyectos, quedando el manual como la única imposición fija.

Las primeras aproximaciones de diseño utilizando el manual se producían en dos canales intermitentes, por una parte se intentaba anticipar cómo se podrían ver los proyectos resultantes al extremar el uso de una o más operaciones para así justificar su selección. Por otra parte, se aplicaban las reglas de alguna de las operaciones del manual para generar "piezas de edificios", elementos reconocibles o icónicos que no replicaran la imagen de arquitectura adorable del manual. En ambos casos, se intentaba justificar una metodología de trabajo en donde el éxito de un resultado se basaba en utilizar las reglas del manual para generar diseños que se opusieran a lo que el manual mecánicamente buscaba generar, proyectos de arquitectura adorable.

Estos primeros diseños evidenciaban la dificultad de articular las operaciones del manual, ya que estas aparecían como acciones independientes agregadas en distintas capas funcionales del proyecto. En este contexto, la necesidad de establecer algún tipo de vínculo entre las operaciones, derivó en varias estrategias de trabajo en los distintos equipos:

- Generar múltiples intentos de proyecto sintetizados en esquemas y croquis rápidos que aceleraran la posible aparición de accidentes o descubrimientos de diseño, y que permitiesen descartar muchas variantes desde sus etapas iniciales, a la vez que generar un repertorio muy básico de elementos para diseñar.

- Trabajar en más de un proyecto de forma simultánea, teniendo cada uno de estos una tipología o destino definido, el desarrollo de estos podían retroalimentarse, y después de un período de desarrollo, descartar el menos favorable.

- Trazar propuestas de diseño que no incluyeran directamente las operaciones del manual, para luego incorporarlas como modificaciones de proyecto. En varios de estos casos, dichas modificaciones no se integraban armónicamente al conjunto o quedaban relegadas a acciones secundarias.

- Estudiar obras y referencias teóricas que compartiesen algunas de las estrategias de diseño de los proyectos en desarrollo. Aunque se entiende que el uso de referentes es una práctica comúnmente extendida al inicio de un proceso de diseño, esta no tuvo relevancia hasta fases mas tardías de trabajo.

En las etapas siguientes, el aprendizaje adquirido a través de estas distintas estrategias de trabajo, pruebas y descartes, así como el uso extensivo de las operaciones del manual derivó en que los proyectos desarrollaran fuertemente dos aspectos, la reinterpretación de las operaciones del manual y la generación de cuerpos argumentativos propios. Respecto del primer punto, las estrategias de diseño más exitosas generaron variantes inesperadas, de las cuales se destacan:

- Repetir muchas veces una de las operaciones para hacer desaparecer la unidad y hacer visible un conjunto o cuerpo mayor radicalmente diferente de este componente singular. Dicha acción hizo que los objetos reiterados se entendiesen como tramas, texturas o masas.

- Reescalar estratégicamente algunos de los componentes de diseño para cambiar su función original y hacer que se 
relacionase de forma distinta con los demás componentes del edificio.

- Cambiar los criterios de posición y articulación de ciertos elementos tectónicos con el fin de alterar radicalmente el rol de estos en el conjunto, desdibujando los límites entre superficies, objetos, vanos, fachadas o cubiertas.

- Hacer aparecer una o más operaciones de manual a través de elementos preexistentes en el sitio del proyecto. Esto consiste en utilizar alguna de estas operaciones y conjugarla con ciertos elementos específicos del lugar, para completar las condiciones que la harían válida acorde a las especificaciones de dicha operación según el manual. Es decir, no se construyen operaciones, sino que se completan a partir de los elementos "así hallados" (Smithson \& Smithson, 2010).

- Modificar las condiciones del lugar de emplazamiento para facilitar o enfatizar la interpretación de las operaciones y su relación con el conjunto del proyecto.

- Buscar puntos de encuentro entre las operaciones del manual, de manera de generar operaciones híbridas, nuevas, que no se reconociesen como la suma de elementos conocidos.

- Explotar los medios expresivos disponibles -y que han sido normados por el manualpara estructurar una narrativa visual que evidencie alguna de las estrategias previamente mencionadas. En este aspecto, más que solo representar, los medios son utilizados como un elemento de retroalimentación para el diseño en curso.

En la medida en que estas estrategias generaron resultados inesperados en el diseño-accidentesrespecto de las expectativas iniciales, cada proyecto adquirió un cuerpo argumentativo propio, que no se basaba en las premisas iniciales de la arquitectura adorable ni en los principios y operaciones descritas en el manual. Esto significó que ningún diseño fuese el resultado de una configuración argumental previa, sino que esta se modificaba constantemente a medida que las estrategias de diseño implementadas articulaban de mejor manera las operaciones del manual.

Considerando que las configuraciones programáticas de cada proyecto, las operaciones y decisiones de diseño cambiaban constantemente, era imposible concebir una sola forma de argumentar el resultado de diseño, y por lo tanto los argumentos nunca dirigieron el diseño, sino más bien articulaban las decisiones de diseño en una narrativa coherente. En este sentido, la estructura argumentativa funcionaba como una conclusión, una extrapolación del diseño, a través del cual se podía entender el proyecto, un cuerpo de ideas independiente que solo aparecía al finalizar el proceso. De esta forma, la argumentación cumplía un rol profundamente pedagógico, ya que no funcionaba como un registro textual que filtrase los proyectos fallidos de los exitosos, sino que cristalizaba los descubrimientos del proceso de diseño, toda vez que permitía sentar posibles caminos de mejora o cambio respecto del estado vigente del proyecto. Ante ese escenario, cada etapa futura de trabajo se retroalimenta de los argumentos, generando un sistema en donde diseño y argumentación se alternan como herramientas de proyecto, produciendo ajustes mutuos, no programados, fracturando la linealidad de un proceso secuencial.

\section{PROYECTOS}

Nuestro equipo de diseño elaboró el proyecto "La Flor", una intervención en el sector de "The Silver Towers" un complejo residencial neoyorquino fuertemente influenciado por las directrices del movimiento moderno, que está conformado por tres torres similares organizadas sobre una placa despejada que se extiende en una plaza dura sobre estacionamientos subterráneos. En este contexto, el proyecto se define por la ejecución de intervenciones mínimas con efectos brutales a través de la utilización de los elementos "así hallados" en el lugar: las torres de departamentos y la grilla regular de columnas del estacionamiento subterráneo, que nunca son intervenidos, ocultados o removidos.

Todas las acciones de diseño se sintetizan en un único elemento: la placa, el plano genérico sobre el que están las torres. Esta superficie que se extiende hacia todo el perímetro de la manzana se convierte en un volumen, una vez que un nuevo perímetro interior de formas contorneadas recorta esta superficie generando dos capas. La capa superior unifica el espacio entre las torres a través de un parque público estrictamente delimitado por los espacios resultantes entre los edificios y el perímetro interior. La capa inferior combina el borde de la manzana y el nivel del subterráneo en una única superficie inclinada, que extiende el suelo de uso público hasta el límite con las calles, fusionándose con las veredas y permitiendo cruzar la manzana. De esta forma, la placa se configura como un límite que separa espacios, usos, escalas y velocidades, pero a la vez las unifica en un solo cuerpo.

El cruce de la manzana se arma a partir del posicionamiento de unidades independientes -locales comerciales en bloques- organizados a partir de relaciones programáticas y de distancia entre ellos, descomponiendo la lectura de ejes de la grilla de columnas subterráneas. Es decir, aparece una forma de recorrer la manzana y definir sus flujos sin las herramientas tradicionales de planificación y diseño urbano; así como hacer aparecer un edificio solo a partir de la reactivación del sitio. Este edificio no se define entonces por la composición de un objeto, sino por la resultante de una serie de intervenciones de cooperación entre los elementos masivos de escala urbana y los componente programáticos de la escala peatonal.

Al examinar la argumentación del proyecto, notamos la ausencia de cualquier referencia al manual de arquitectura adorable o al cliché que lo justificaba. En efecto, en la etapa final, ninguna de las múltiples propuestas de 
diseño iniciales perduró o se respetó para las etapas siguientes. Las operaciones descritas en el manual aparecieron en los proyectos no como un perjuicio, sino como articuladores o compositores de estos, y el fundamento inicial de explotar los clichés de la arquitectura japonesa "adorable" no ayudaba a explicar ni fundamentar los proyectos, ya que estos desarrollaron un cuerpo argumental propio.

Que cada proyecto lograse configurar una estructura argumental propia alejada totalmente del inicio revela dos aspectos, que la metodología de trabajo perdura como único aspecto invariable durante el proceso de desarrollo (utilización del manual como base para todas las decisiones de diseño), es decir, que los argumentos iniciales definen el método de trabajo, y no anticipan el resultado; y que un proyecto puede evolucionar desde cualquier premisa, sea esta planificada o arbitraria.

Quizás este último punto es el más relevante de esta experiencia de diseño, que la premisa inicial puede ser totalmente arbitraria, que un proyecto puede surgir desde cualquier escenario, sea este favorable o no a lo que uno idealmente quisiera para diseñar. Fue totalmente irrelevante si el manual de clichés estaba basado en técnicas de diseño arquitectónico japonés o de otro país, si eran diez o más reglas o si se hubiese considerado otro cliché para justificar todo; todos estos aspectos podrían haber sido totalmente diferentes, ya que el valor de esta metodología radica en hacernos conscientes de los límites sobre los cuales podemos operar; siendo estos escogidos, sistematizados y definidos por nosotros mismos acorde a un conjunto de datos de los que todos tuviésemos conocimiento (decenas de obras de referencia). Mientras dichos reglamentos podrían haber sido dictados por un externo, el solo hecho que los mismos estudiantes hayamos elegido sobre qué trabajar, qué reglas seguir y cómo trabajar con estas, nos hacía responsables de sus resultados.

Para poner a prueba esta conclusión, apliqué la metodología del manual de arquitectura adorable para diseñar un proyecto totalmente
4. Proyecto de Vivienda Premio Todoobras 2014 Créditos: Claudio Palavecino.

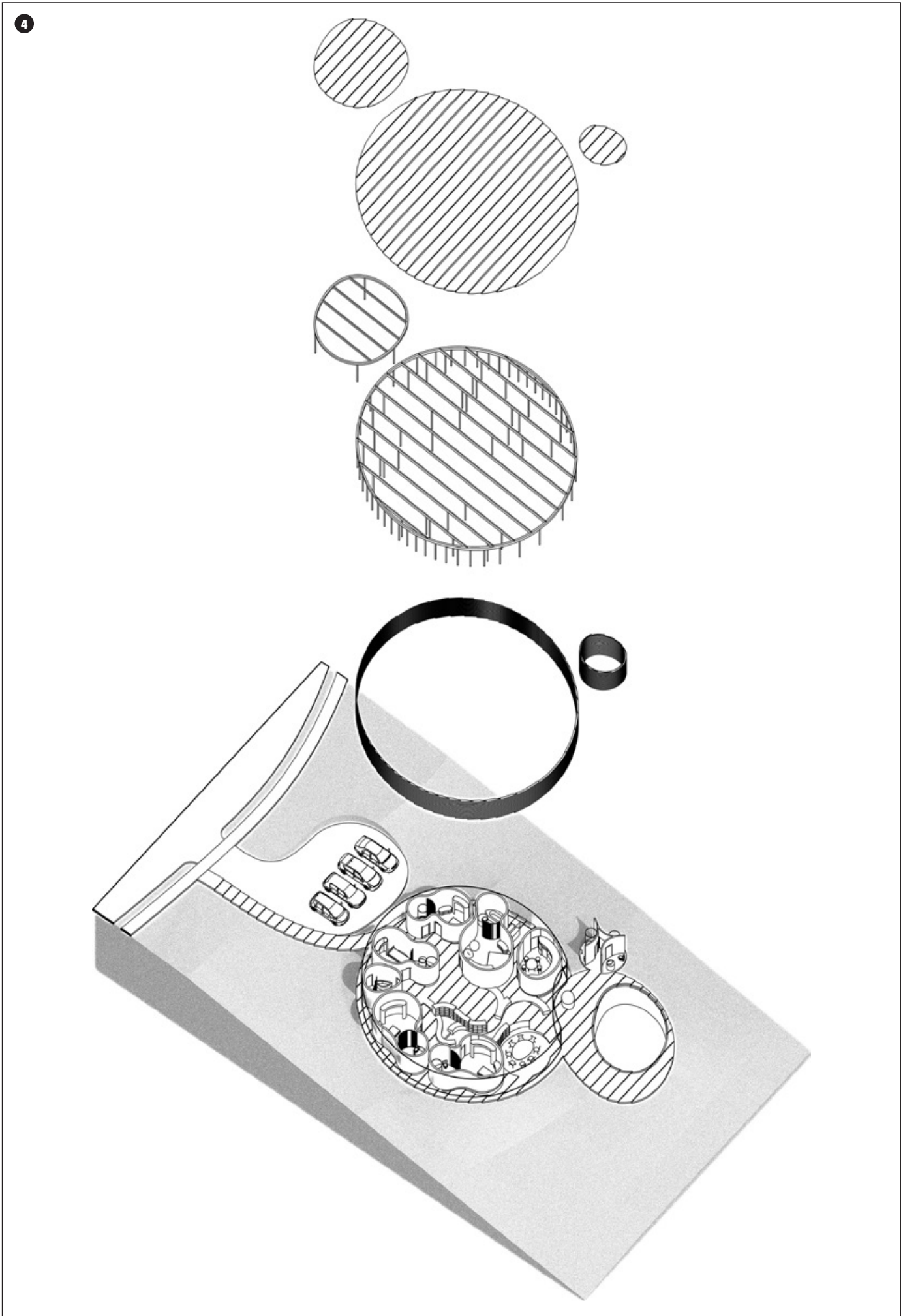


diferente un par de meses después. Este era una propuesta para un reciente concurso nacional que solicitaba el diseño de una vivienda unifamiliar en el sector oriente de Santiago, fijando sus bases según ciertos objetivos de construcción de bajo impacto ambiental (Premio Todoobras de Vivienda Sustentable 2014). Para plantear la propuesta, decidí utilizar las mismas operaciones descritas en el manual que ya antes había usado, agregando otras tres operaciones intencionalmente arbitrarias, que la planta del proyecto fuese circular, la imposición de escaleras para circular entre las distintas habitaciones de la vivienda, y la ausencia de esquinas y rincones. Estas reglas se combinarían con las otras ya antes definidas para definir un marco de operaciones nuevo para diseñar esta vivienda, totalmente fuera de todo ideal de arquitectura adorable y en un contexto diametralmente opuesto al original (aquí se debía diseñar una casa, mientras que el proyecto académico devino en propuestas de edificios públicos en distintos lugares de Nueva York).

La vivienda propuesta, se concibe a partir de dos configuraciones programáticas opuestas, por una parte unidades herméticas que albergan las habitaciones y zonas húmedas, cuyo mobiliario, tamaño y distribución obedece a actividades específicas de cada una. Por otra parte, un espacio multiprogramático alberga todas las actividades de uso compartido en un único espacio genérico, sin divisiones ni zonas de uso, resultante de la superficie libre entre los cerramientos de las habitaciones y los límites de la vivienda. Este conjunto se organiza en una única superficie circular, en la que no existen circulaciones definidas ni ejes distribuidores de espacios -acentuado por la curvatura de todos los volúmenes y la ausencia total de rincones-, en el que el espacio común actúa como contenedor y articulador de todas las actividades.

Este espacio compartido adopta el perfil de la pendiente del terreno, convirtiendo el plano de actividades comunes en una sucesión de múltiples niveles sin divisiones entre pisos, haciendo que la superficie de la casa sea en sí una gran escalera. Esta "escalera", que recorre ininterrumpidamente los espacios exteriores e interiores del proyecto, entra en contacto con los muros y cerramientos interiores, generando espacios de distintas calidades y potenciales usos para las actividades del hogar a partir de las variaciones de altura y tamaño de los escalones -que se confunden con el mobiliario-, así como la proximidad con las habitaciones y las fachadas interiores, que se arman fusionando toda la envolvente de la vivienda con el paisaje en una sola textura, que varía en luz, sombra e intensidad a través de las horas del día.

Más allá que el proyecto fuese uno de los premiados, la interpretación del jurado y los fundamentos con que fue presentado no delataban su origen en un manual o en las premisas arbitrarias ya mencionadas, porque al igual que en la experiencia del "Diccionario de Ideas Recibidas", el proyecto adquirió un cuerpo argumental independiente a medida que descubría nuevas relaciones y combinaciones entre las operaciones sobre las cuales se basaba. Las bases arbitrarias originales solo configuran una estructura para diseñar que debe desaparecer para hacer visible el resultado final, de forma similar a los andamios de una construcción (Walker, 2013).

\section{ARBITRARIEDAD ÚTIL}

Las reglas arbitrarias con las que se diseñaron estos proyectos no deben entenderse como meras imposiciones aleatorias que desajustan las convenciones del proceso de diseño, sino como herramientas funcionales. Estas restricciones tienen que estar calibradas para no ser lo suficiente obtusas como para obstruir cualquier opción de diseño, ni lo suficientemente permisivas como para permitir cualquier decisión, ni lo suficientemente predecible como para conducir a soluciones recurrentes (Walker, 2013). Estas reglas son útiles porque generan las condiciones para formular problemas nuevos y resultados no planificados en beneficio del proyecto, llevando el proceso de diseño al campo de lo inexplorado, lo que impide anticipar cualquier posible resultado, y focaliza el proceso de diseño en explotar las bases sobre las cuales se trabaja. Esto implica desligar el diseño de los lugares comunes que se utilizan para fundamentar los proyectos, y hacer que los argumentos se constituyan como una síntesis de los descubrimientos del proceso.

De la misma forma que las reglas arbitrarias fueron funcionales, también lo fue la utilización de un cliché de la arquitectura como inicio de proyecto. Ambos exigen ser entendidos con total claridad porque constituyen el espectro único de conocimiento sobre el cual operar, sin embargo ambos se calibran con distintos grados de ajuste. Mientras las operaciones de diseño fueron explicitadas con extrema precisión para ser llevadas a sus límites, el cliché se explica a partir de principios generales, lo que podría parecer contradictorio, ya que el conocimiento pleno del cliché justificaría el consenso en torno a las reglas de diseño. Sin embargo, esto no es más que una constatación de la arbitrariedad necesaria para poder avanzar en las distintas etapas de trabajo, porque las reglas de diseño surgen solo desde la necesidad de quienes elaboraron el manual, una necesidad que en nada justifica el cliché, que solo se reconoce por su uso reiterativo y agotado. Por esto, el cliché "desaparece" una vez que las reglas definen el manual de diseño, que a la vez "desaparecen" cuando surge el proyecto elaborado a partir de este manual, que a la vez "desaparece" al generarse un proyecto nuevo a partir del cuestionamiento del original.

En vez de acumular estratos de argumentos que rigidizan la evolución del proyecto, cada etapa proyectual y los argumentos que la sostenían sirvieron solo como un precedente para la generación una etapa posterior nueva. Este procedimiento puede de alguna forma homologarse a la necesidad de plantear nuevos paradigmas en la arquitectura contemporánea declarando retrógrados y obstructivos aquellos aspectos que definían la autonomía disciplinar que validó los paradigmas moderno y posmoderno, pese a que esto es solo posible 
cuando se opera desde el conocimiento y control de estos (Hays, 2003).

El enfoque pedagógico del taller del "Diccionario de Ideas Recibidas" fue una constatación permanente de cómo el proceso de proyecto -si pretende promover la inventiva y crear conocimiento nuevo a través del diseño- se sostiene en el cambio constante, la transmutación de un proyecto a otro sobre la base de los descubrimientos en el diseño, aun si eso significa desmoronar lo que se esperaba de un proyecto. Fue un ejercicio de constante extracción de conocimiento para proyectar a partir de la intensificación y explotación de lo que se conoce, ejecutado con riguroso respeto de las reglas arbitrarias consensuadas, ya que estas visibilizan el encuentro con los clichés, lugares comunes y convenciones que se quieren evitar.

A través de este proceso no solo se produjo una aproximación crítica a la forma en que desarrollamos proyectos, sino una revalorización de todo el legado histórico, tecnológico y teórico de la arquitectura como precedente de todo proyecto futuro. En palabras de Kenneth Frampton (2002), la arquitectura es por definición anacrónica ya que se posiciona simultáneamente tanto el pasado como el presente a través de una mediación política entre la innovación y la tradición (Frampton, 2002, p. 18). En ese sentido, la experiencia del taller explicada en este artículo, revela cómo este ejercicio proyectual comenzó desde un cuestionamiento crítico de lo que conocemos a través del figura del cliché, concientizándonos de cómo estos elementos tectónicos se definieron, cómo fueron usados y simbolizados a través de la historia de la arquitectura para finalmente convertirlos en el marco operativo -reglas de diseño en un manual-sobre el cual se desprendió un estrato nuevo de conocimiento, un producto que construye desde lo sabido, pero se sostiene por argumentos propios.

\section{REFERENCIAS}

Allen, S. (2009). From Object to Field: Field Conditions in Architecture and Urbanism. En S. Allen (Ed.), Practice: Architecture, Technique and Representation (pp. 216-241), Oxford: Routledge.

Eisenman, P. (2008). Diez Edificios Canónicos 19502000. New York: Rizzoli.
Frampton, K. (2002). Labour, Work and Architecture Collected Essays on Architecture and Design. New York: Phaidon.

Hays, M. (2003). The Autonomy Effect. En G. Damiani (Ed.), Bernard Tschumi (pp. 6-17), London: Thames \& Hudson.

Momoyo, K., Kuroda, J. \& Tsukamoto Y. (2001). Made in Tokyo. Tokio: Kaijima Institute Publishing Co.

Mumford, E. (1995). The "tower in a park" in America: theory and practice, 1920-1960. Planning Perspectives, 1(10), 17-41.

Nuijsink, C. (2012). How to make a Japanese House. Rotterdam: Nai Publishers.

Smithson, P. \& Smithson, A. (2010). Lo "así hallado" y "lo hallado". En E. Walker (Ed.), Lo Ordinario (pp. 93-99). Barcelona: Gustavo Gili.

Tschumi, B. (1994). Architecture and Disjunction. Cambridge: The MIT Press.

Tschumi, B. \& Walker, E. (2004). Avant-Propos: Bernard Tschumi in Conversation with Enrique Walker. Grey Room, (17), 118-126.

Walker, E. (2010). Compendium. En Tsukamoto Y. y Kaijima M. (Eds.), The Architectures of Atelier Bow-Wow: Behaviorology, (pp. 345-349). New York: Rizzoli.

Walker, E. (2013). Calligram. En M. Fornabai (Ed.), Ink or "V is for Vermilion as described by Vitruvius" An A to Z of Ink in Architecture (pp. 12-13). New York: GSAPP Books. 